\title{
Persistent photoconductivity in InAsN/InGaAs quantum wells
}

\author{
J.C. Fan ${ }^{\mathrm{a}, *}$, Y.F. Chen ${ }^{\mathrm{b}}$ \\ ${ }^{a}$ Department of Electrical Engineering, Da-Yeh University, Chang-Hwa, Taiwan \\ ${ }^{\mathrm{b}}$ Department of Physics, National Taiwan University, Taipei, Taiwan
}

Received 17 January 2008; accepted 10 March 2008 by M. Skolnick

Available online 14 March 2008

\begin{abstract}
Optical properties of InAsN/InGaAs quantum wells on InP grown by gas source molecular beam epitaxy have been investigated by photoconductivity measurements. It is found that the persistent photoconductivity (PPC) does exist in this nitrogen compound material. Through a detailed study of the dependence of the PPC effect on excitation photon energy, temperature, decay kinetics and nitrogen composition, we point out that the origin of the PPC effect arises from the metastability of deep level centers. In different charge states, the defect centers can undergo a large lattice relaxation which creates an energy barrier to prevent the photoexcited carriers from returning to the original states, and thus the PPC occurs. We find that the obtained energy barrier increases with nitrogen composition.
\end{abstract}

(c) 2008 Elsevier Ltd. All rights reserved.

PACS: 72.20.Jv; 72.40.+w; 72.80.Ey

Keywords: A. Quantum well; A. Semiconductors; D. Photoluminescence; D. Photoconductivity

Low nitrogen content III-V alloy semiconductors have recently attracted much attention [1-8] due to their interesting physical properties and potential applications of optoelectronic devices. Especially, it leads to the development of technical application of long-wavelength laser diodes. For instance, InGaAsN grown on GaAs substrate is suitable for longwavelength laser diodes at $1.3 \mu \mathrm{m}$ [1-3], and InNAsP grown on InP substrate for $1.55 \mu \mathrm{m}$ laser [4]. Both of them are very important for optical fiber communication. In the mid-infrared 2-5 $\mu \mathrm{m}$ optoelectronic devices [5], InAsN alloy could be a very promising material. By the advantage of highly strained multiquantum well, it is possible to fabricate good quality material and push the laser emission of InAsN based devices to the desired wavelength. However, most of the previous reports were mainly concentrated on the dependence of the InAsN band gap on $\mathrm{N}$ composition [6-9]. To the best of our knowledge, the study of optoelectric properties is rather limited for InAsN material. In this paper, we present the result of optical studies on InAsN/InGaAs quantum wells.

\footnotetext{
* Corresponding address: Department of Electrical Engineering, Da-Yeh University, No. 112, Shanjiao Road, 515 Chang-Hwa, Taiwan. Tel.: +886 4 8511888; fax: +886 48511245 .

E-mail address: fanjc@ mail.dyu.edu.tw (J.C. Fan).
}

Except the confirmation of the incorporation of nitrogen in InAs and the effect of quantum confinement, it is found that the persistent photoconductivity does exist in InAsN quantum wells. The PPC effect is the photoenhanced conductivity that persists for a very long period of time after the termination of photoexcitation. This peculiar phenomenon has been observed in several important semiconductors, and the related studies are very useful for the understanding of defect properties [10-13]. Through a detailed study, our results reveal that the PPC effects in InAsN quantum well can be attributed to the metastability of deep defects due to large local strain from the nitrogen incorporation. In different charge states, the relaxation of local strain can create an energy barrier to prevent the recombination of photoexcited electron-hole pairs, and thus the PPC occurs.

The samples were grown on semi-insulating (100) InP substrates using a VG V-80H gas source molecular beam epitaxy (GSMBE) system. Element In, Ga, and thermally cracked $\mathrm{AsH}_{3}$ and $\mathrm{PH}_{3}$ sources were used. An EPI UNI-bulb rf plasma source operated at a radio frequency of $13.56 \mathrm{MHz}$ was used to generate active $\mathrm{N}$ species. The rf power for generating $\mathrm{N}$ species was $300 \mathrm{~W}$. After the thermal cleaning of the InP substrate at $500{ }^{\circ} \mathrm{C}$ under $\mathrm{P}_{2}$ flux, the brightness mode $\mathrm{H}_{2}$ plasma was first ignited and a $0.1 \mu \mathrm{m}$-thick InP layer was then 
grown as a buffer layer at $450{ }^{\circ} \mathrm{C}$. Some reports indicated that atomic hydrogen can enhance the removal of surface oxide and passivate some impurities and defects [5,6]. Then, the growth was interrupted and the substrate temperature was reduced to $400{ }^{\circ} \mathrm{C}$ for $\mathrm{InGaAs} / \mathrm{InAs}(\mathrm{N}) / \mathrm{InGaAs}$ single quantum well (SQW) growth. The SQW structure layer is comprised of two $100 \mathrm{~nm}$-thick $\operatorname{In}_{0.53} \mathrm{Ga}_{0.47}$ As barriers and a $30 \AA$-thick InAs(N) well. The growth rate for $\operatorname{In} A s(N)$ was about $1 \mu \mathrm{m} / \mathrm{h}$. There were no interruptions at the heterointerfaces of the SQW. The gas line of the first SQW interface was starting. During the gas switching, the plasma was still maintained at high brightness mode. The rf power was turned off when the growth of the SQW was finished. The beam equivalent pressure (BEP) of the $\mathrm{N}$ source during the growth of InAsN SQW was around $10^{-5}$ to $10^{-4}$ mbar, depending on the nitrogen flow rates. The exact nitrogen content in InAsN SQW was unknown, because the well-thickness is too thin to be determined by X-ray spectrum measurements. Instead of the $\mathrm{N}$ composition, we use the BEP to divide the different samples. Finally, a $0.1 \mu \mathrm{m}$-thick InP cap layer was over grown on the SQW.

The photoconductivity measurements were carried out in a liquid-nitrogen-bath cryostat in which the varied temperatures ( $T=77 \mathrm{~K}-300 \mathrm{~K})$ were controlled by an ITC4 temperature controller. Ohmic contacts were formed by depositing indium drops at the four corners of the samples, and annealing the contacts at $400{ }^{\circ} \mathrm{C}$ for $10 \mathrm{~s}$. A tungsten lamp dispersed by a $550 \mathrm{M}$ monochromator was used as the photoexcitation light source. A constant current was supplied to the sample by a Keithley 236 source measure unit. The conductivity signal was detected as a change in the voltage drop across the sample using a lock-in amplifier. For the PPC measurement, the samples were cooled in darkness to a required temperature and then exposed to light for a period of $20 \mathrm{~min}$. The light was then turned off, and the system was allowed to relax for several hours. The data obtained at different conditions were taken in such a way that after a measurement the system was gradually heated up to room temperature to recover the sample to its initial state and then cooled down again in darkness to the temperature of measurement. This is to ensure that the data obtained for each measurement has the same initial condition.

The dependence of the PL spectra of the InAsN SQWs on nitrogen flow rate is shown in Fig. 1. As can be seen, the PL peak position decreases with increasing nitrogen flux. This indicates a bowing effect of the bandgap due to the incorporation of nitrogen in this alloy system. The PL intensity degrades very rapidly with increasing of nitrogen concentration. These results indicate the presence of high concentration nonradiative centers. Since there is a very large atomic-size difference between $\mathrm{N}$ and As, the large local strain from the nitrogen incorporation in InAs crystal could result in inferior crystallinity. Fig. 2 shows the dependence of the InAsN SQW PL peak energy on the nitrogen flux. The bowing parameter is estimated to be about $15 \mathrm{eV}$ in our samples, if we assume the nitrogen content is linearly proportional to the nitrogen flow rate, and the nitrogen content for the sample deposited with nitrogen flux of $9 \times 10^{-5}$ mbar is around

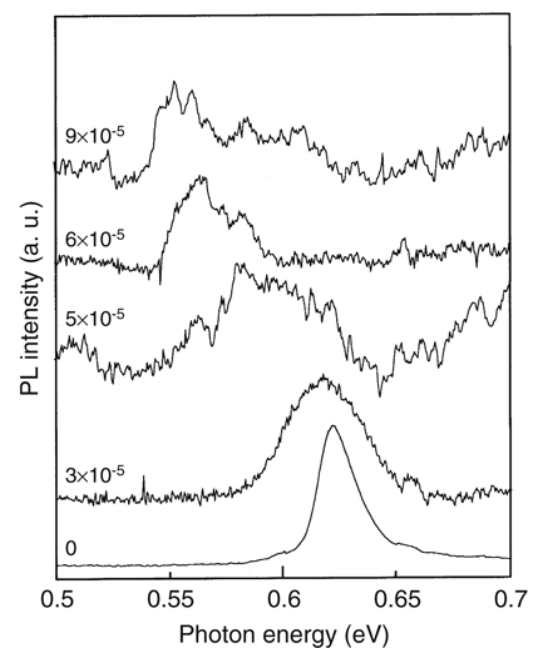

Fig. 1. Photoluminescence spectra of InAsN/InGaAs SQW structures as a function of nitrogen flux at $77 \mathrm{~K}$. The gas pressure is in mbar.

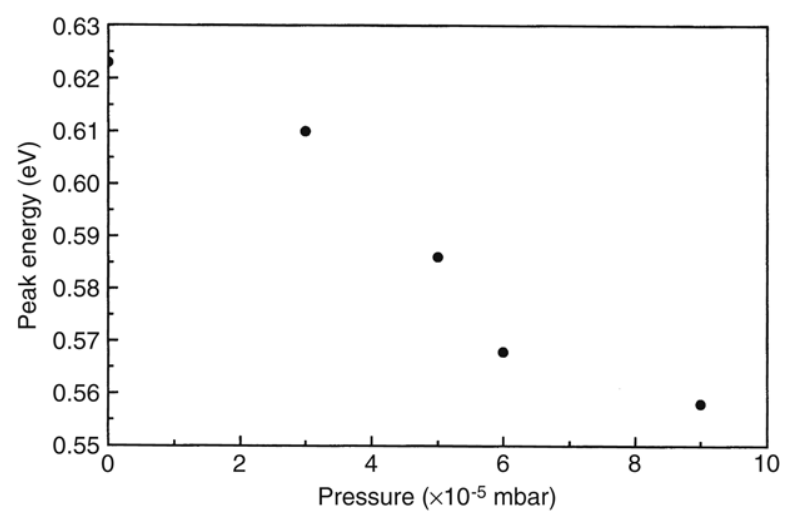

Fig. 2. Dependence of the photoluminescence peak position on nitrogen flux for InAsN single quantum well.

$0.4 \%$ [14]. This value is in good agreement with the low nitrogen content in other III-V compound systems [15-18].

Fig. 3 shows the resulting photoconductivity (PC) spectrum of the InAsN SQW with the lowest nitrogen content at different temperature. The clearly identified peak exhibits a redshift as temperature increases. Comparing the PL and PC peak energy at temperature $77 \mathrm{~K}$, there exists a Stokes' shift in the InAsN SQW. This behavior may indicate that the emission arises from localized states which are due to the random nature of InAsN alloy. In addition, because of the large difference in atomic sizes and electronegativities of $\mathrm{N}$ and As, it causes the phase segregation in InAsN alloys, i.e. InAs and $\mathrm{InN}$ phases. This inhomogeneity can also produce potential fluctuations and create the localized states for the observed emission. Of course, we cannot rule out the possibility of the influence of piezoelectric effect on the Stokes' shift. A rather interesting behavior in Fig. 3 is that starting from $77 \mathrm{~K}$ the PC signal increases with increasing temperature, and then it decreases with increasing temperature when the temperature exceeds $140 \mathrm{~K}$. These characteristic properties appear conspicuously in the fluctuation of $\mathrm{PC}$ signals after the temperature at $140 \mathrm{~K}$. This behavior clearly demonstrates the important role of localized defects in the determination of optical properties 


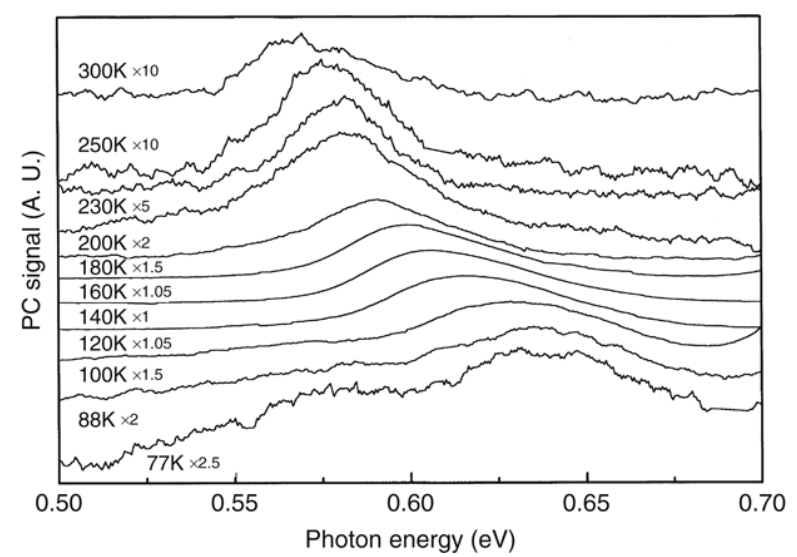

Fig. 3. Temperature-dependent photoconductivity spectra of InAsN single quantum well deposited with nitrogen flux of $3 \times 10^{-5} \mathrm{mbar}$.

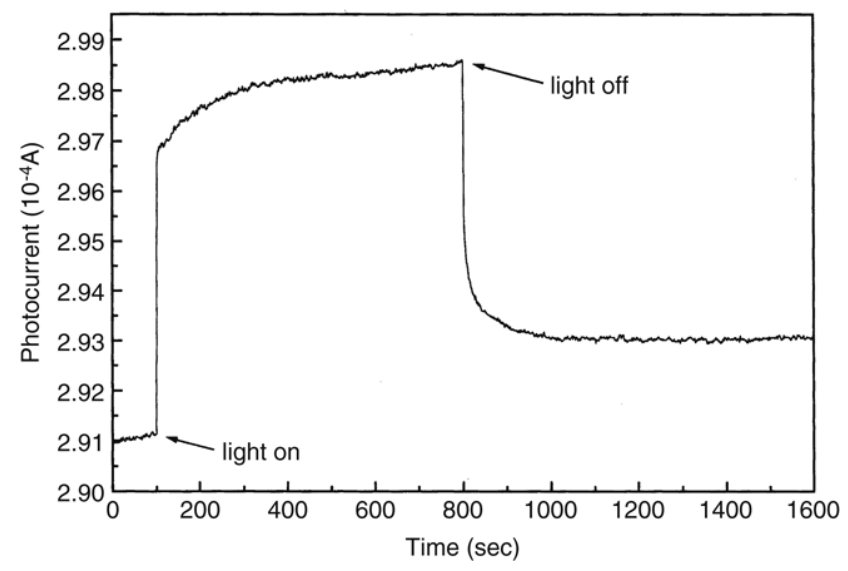

Fig. 4. Typical buildup and decay behavior of persistent photoconductivity in InAsN SQW with nitrogen flux $3 \times 10^{-5}$ mbar at $77 \mathrm{~K}$.

in InAsN quantum wells. At low temperatures the photoexcited carriers are trapped in the localized states which are less mobile and lead to the smaller PC signal. With increasing temperature, the trapped carriers can be thermally excited to extended states and the PC signal is enhanced.

In the study of the PC measurements, we discovered the existence of PPC effect in InAsN/InGaAs quantum wells. Fig. 4 shows a typical photoconductivity build up and decay of the InAsN SQW with the lowest nitrogen content at temperature $77 \mathrm{~K}$. We can see that the photocurrent increases gradually after the samples are exposed to light and that the photoinduced current can persist for a very long period of time after removing the light. There are several different mechanisms that can cause PPC effect as shown in previous reports [19-26]. In order to examine the exact mechanism responsible for the PPC in InAsN/InGaAs quantum wells, we have performed the measurement at different pumping photon energy. It is found that the PPC has the same behavior for the pumping photon energy above and below the barrier band gap. We therefore can exclude the possibility that the PPC arises from the charge transfer between the barrier and well layers. The PPC occurs because of the photoexcitation in the well region.

To further explore the mechanism of the PPC effect in InAsN/InGaAs quantum wells, we measure the PPC decay

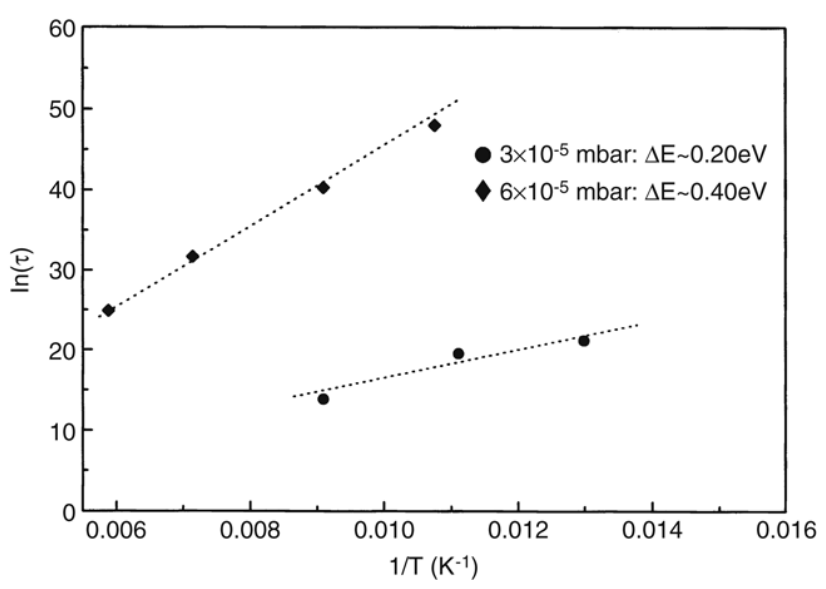

Fig. 5. The Arrhenius plot of PPC decay time constant $\ln \tau$ vs $1 / T$ for different nitrogen fluxes.

kinetics. Investigation in decay kinetics has led to a deeper understanding of the carrier relaxation and the metastability of crystal defects [20-26]. It is found that the decay kinetics of the PPC in InAsN/InGaAs quantum wells can be described by a stretched-exponential function

$I_{\mathrm{PPC}}(t)=I_{\mathrm{PPC}}(0) \exp \left[-(t / \tau)^{\beta}\right]$,

where $I_{\mathrm{PPC}}(0)$ is the PC buildup level at the moment of light excitation being removed, $\tau$ is the PPC decay time constant, and $\beta$ is the decay exponent. Usually, the observation of stretched-exponential decay in a material suggests the existence of random nature in the origin of decay process since Eq. (1) has been used to describe many relaxation phenomena in amorphous systems [19-26].

Fig. 5 shows that the Arrhenius plot of the PPC decay time constant for the InAsN quantum wells with different nitrogen content. We can see that $\tau$ decreases with temperature following an activated behavior. The temperature dependence of $\tau$ can be described by $\tau=\tau_{0} \exp [\Delta E / k T]$, where $\Delta E$ is the thermal activation energy, and $\tau_{0}$ is a constant. We obtained that the values of $\Delta E$ are $0.2 \mathrm{eV}$ and $0.4 \mathrm{eV}$ corresponding to the nitrogen pressure at 3 and $6 \times 10^{-5}$ mbar, respectively. Such a large thermal activation energy can be used to rule out the possibility that the PPC effect arises from band-tail localized states caused by the potential fluctuations of alloy disorder or phase segregation. It is because of the fact that the observed Stokes' shift between PL and PC spectrum is much smaller than the activation energy obtained here. We therefore suggest that the PPC effect is associated with the deep-level traps, which undergo a large lattice relaxation when the defect traps are in different charge states. In this model, the lattice relaxation can create an energy barrier to prevent the photoexcited carrier from recombination, and hence the PPC occurs. This mechanism has been used to interpret PPC effects in many III-V semiconductors [20-23]. It is worth noting that the thermal activation energy increases with increasing nitrogen contents in our study. This behavior indicates that the trapping defect is related to $\mathrm{N}$ incorporation. The different nitrogen contents can modify the atomic configuration near the trapping defects and cause different degree of lattice relaxation. 
In summary, we have investigated the optical properties of InAsN/InGaAs quantum wells on InP grown by gas source molecular beam epitaxy. In addition to the confirmation of the incorporation of nitrogen in InAs and the quantum confinement, we observed the existence of PPC effect. Through a detailed study of the PPC effect, we pointed out that the PPC effect arises form the large lattice relaxation of defect centers. In different charge states, the defect centers in different atomic configurations create an energy barrier to prevent the photoexcited carriers from recombination and causing the PPC effect. We also found that the energy barrier increases with the incorporation of nitrogen content.

\section{Acknowledgments}

This work was partially supported by the National Science Council and Ministry of Education of the Republic of China.

\section{References}

[1] M. Kondow, K. Uomi, T. Kitatani, S. Watahiki, Y. Yaxawa, J. Cryst. Growth 164 (1996) 175.

[2] M. Kondow, T. Kitatani, S. Nakatsuka, M.C. Larson, K. Nakahara, Y. Yaxawa, M. Okai, K. Uomi, IEEE J. Sel. Top. Quantum Electron. 3 (1997) 719.

[3] M. Motyka, R. Kudrawiec, G. Sek, J. Misiewicz, D. Bisping, B. Marguardt, A. Forchel, M. Fischer, Appl. Phys. Lett. 90 (2007) 221112

[4] W.G. Bi, C.W. Tu, Appl. Phys. Lett. 72 (1998) 1161.

[5] D.K. Shih, H.H. Li, Y.H. Lin, Electron. Lett. 37 (2001) 1342.
[6] S.R. Kurtz, A.A. Allerman, E.D. Jones, J.M. Gee, J.J. Banas, B.E. Hammons, Appl. Phys. Lett. 74 (1999) 729.

[7] R. Beresford, K.S. Stevens, A.F. Schwartzman, J. Vac. Sci. Technol. B 16 (1998) 1293.

[8] J. Wagner, K. Köhler, P. Ganser, M. Maier, Appl. Phys. Lett. 87 (2005) 051913.

[9] M. Motyka, R. Kudrawiec, G SeK, J. Misiewicz, D. Bisping, B. Marquardt, A. Forchel, M. Fischer, J. Appl. Phys. 101 (2007) 113539.

[10] D.V. Lang, R.A. Logan, Phys. Rev. Lett. 39 (1977) 635.

[11] P.M. Mooney, J. Appl. Phys. 67 (1990) R1.

[12] H.X. Jiang, J.Y. Lin, Phys. Rev. Lett. 57 (1990) 873.

[13] L.H. Chu, Y.F. Chen, D.C. Chang, C.Y. Chang, J. Phys. Condens. Matter 7 (1995) 4525.

[14] J.S. Wang, H.H. Lin, J. Vac, Sci. Technol. B 17 (1999) 1997.

[15] S. Sakai, Y. Ueta, Y. Terauchi, Jpn. J. Appl. Phys. 32 (1993) 4413.

[16] J.N. Baillargeon, K.Y. Cheng, G.E. Hofler, P.J. Pearah, K.C. Hsieh, Appl. Phys. Lett. 60 (1992) 2540.

[17] M. Kondow, K. Uomi, K. Hosomi, T. Mozume, Jpn. J. Appl. Phys. 33 (1994) L1056.

[18] G. Pozina, I. Ivanov, B. Monemar, J. Appl. Phys. 84 (1998) 3830.

[19] A. Dissanayake, M. Elahi, H.X. Jiang, J.Y. Lin, Phys. Rev. B 45 (1992) 13996.

[20] J.Z. Li, J.Y. Lin, H.X. Jiang, J.F. Geisz, S.R. Kurtz, Appl. Phys. Lett. 75 (1999) 1899.

[21] J.Y. Lin, A. Dissanayake, G. Brown, H.X. Jiang, Phys. Rev. B 42 (1990) 5855 .

[22] W. Götz, N.M. Johnson, M.D. Bremser, Q.F. Davis, Appl. Phys. Lett. 69 (1996) 2379.

[23] H.M. Chen, Y.F. Chen, M.C. Lee, M.S. Feng, J. Appl. Phys. 82 (1997) 899.

[24] D.V. Lang, R.A. Logan, Phys. Rev. B 19 (1979) 1015.

[25] M.K. Sheinkman, A.Y. Shik, Sov. Phys. Semicond. 10 (1976) 128

[26] D.E. Theodorou, H.J. Queisser, Appl. Phys. 23 (1980) 121. 